\title{
On the hydrophobic chains effect on critical micelle concentration of cationic gemini surfactants using molecular connectivity indices
}

\author{
Anna Mozrzymas ${ }^{1}$
}

Received: 31 January 2020 / Accepted: 12 March 2020 / Published online: 28 April 2020

(c) The Author(s) 2020

\begin{abstract}
The influence of the structure of hydrophobic tail chains on the critical micelle concentration of cationic gemini surfactants, using only the molecular connectivity indices, has been investigated in this work. The best model obtained shows that the relationship between the logarithm of critical micelle concentration and the alkyl chains length is parabolic. The formula has been derived for compounds with the same head groups and the same, medium length, spacer but with various hydrocarbon tail chains. Good-quality QSPR model obtained can be used to predict the critical micelle concentration value of structurally similar gemini surfactants as well as to design the structure of the hydrophobic tail chains to obtain new molecules more active in micelle formation.
\end{abstract}

Graphic abstract

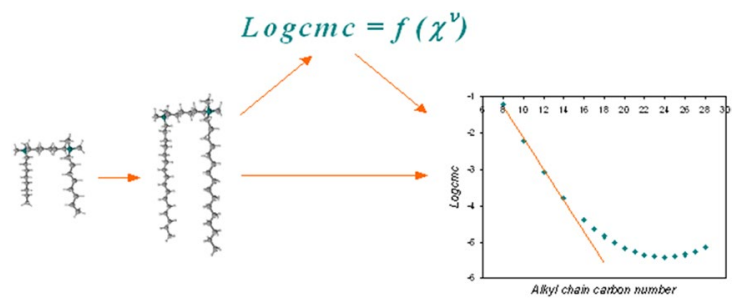

Keywords Cationic surfactants · Chains · Critical micelle concentration · QSPR · Topological descriptors

\section{Introduction}

One of the most important parameter characterising a surfactant is its critical micelle concentration, in short $\mathrm{cmc}$. Among the factors known to affect the $c m c$ value in aqueous solution is the structure of the surfactant [1]. Therefore, as shown in many articles [2-18], the critical micelle concentration can be predicted directly from the structure of the molecule by quantitative structure_-property relationship analysis, in short QSPR. The first correlation was given by Klevens [19] who empirically found that the logarithm of cmc linearly decreases with the increase in the number of

Anna Mozrzymas

anna.mozrzymas@upwr.edu.pl

1 Department of Physics and Biophysics, Wrocław University of Environmental and Life Sciences, ul. Norwida 25,

50-375 Wrocław, Poland carbon atoms in the surfactant alkyl chain. All surfactants have an amphiphilic structure. They contain both hydrophobic groups called tails and hydrophilic ones which are called heads. The conventional monomeric surfactants are formed from one tail and one head while the gemini surfactants consist of two hydrophobic groups and two hydrophilic heads connected by the spacer group. In the case of the conventional surfactants, the logarithm of $\mathrm{cmc}$ is a linear function of alkyl chain carbon number to at least sixteenth carbon atoms while the logarithm of $\mathrm{cmc}$ of gemini surfactants start to deviate more and more from this linear relationship at number of carbon atoms greater than 14 [1]. For some cationic gemini surfactants, this deviation from linearity becomes an increase in value of logarithm of $\mathrm{cmc}$. In the aqueous solution at room temperature, the minimum was observed for several series of cationic gemini surfactants having the spacer groups or tails of different nature and flexibility 
[20-22]. In the case of cationic gemini surfactants with only straight hydrocarbon chains (tails and spacer), according to the author's best knowledge, the minimum logarithm $\mathrm{cmc}$ value was not observed. However, in the aqueous solution at room temperature, the critical micelle concentrations were measured using surfactants with the maximum alkyl chains length of 18 carbon atoms.

Therefore, the author of this paper decided to theoretically analyse the effect of the structure of the hydrophobic tail chains of cationic gemini surfactant on $c m c$ using, as in the previous papers $[4,5,7,10,11]$, the molecular connectivity indices [23] only. The simple theoretical model, that will allow to study the effect of the hydrocarbon chains structure modifications on the value of the critical micelle concentration, has been derived for compounds with fixed heads and fixed medium spacer length but with different hydrophobic tail chains.

\section{Results and discussion}

To find and study the dependence of the critical micelle concentration on the structure of the hydrophobic gemini surfactants tail chains, the surfactants with fixed heads and spacer group and having only hydrocarbon chains were chosen. The structures of all considered molecules are shown in the "Methods" section.

Based on the literature data of $\mathrm{cmc}$ [24-27] and values of molecular connectivity indices [7] of training set compounds 1-10 (see "Methods"), using the polynomial regression analysis and stepwise method, two second-order regression models have been obtained. For each model, the search of the best equation consisted of two steps. The first step is shown in Table 1.

As shown in Table 1, the highest correlation coefficient values are for the relationships containing the second-order valence molecular connectivity index $\left({ }^{2} \chi^{v}\right)$ and also the first-order valence molecular connectivity index $\left({ }^{1} \chi^{v}\right)$. These indices define Model 1 and Model 2, respectively. The second step for each model is shown in Table 2.

As shown in Table 2, the best equations were obtained using in addition the square of the following indices: the second-order valence molecular connectivity index $\left[\left({ }^{2} \chi^{v}\right)^{2}\right]$ in Model 1 and the first-order valence molecular connectivity index $\left[\left({ }^{1} \chi^{v}\right)^{2}\right]$ in Model 2 . The correlation formulas (Models 1-2) are as follows:

Model 1:

$$
\begin{aligned}
\log _{10} c m c= & (10.14849 \pm 1.94143) \\
& -(1.39052 \pm 0.28227) \cdot{ }^{2} \chi^{v} \\
& +(0.03060 \pm 0.01008) \cdot\left({ }^{2} \chi^{v}\right)^{2} .
\end{aligned}
$$

Model 2:

$$
\begin{aligned}
\log _{10} c m c= & (8.38205 \pm 1.37961) \\
& -(0.95624 \pm 0.15916) \cdot{ }^{1} \chi^{\nu} \\
& +(0.01656 \pm 0.00449) \cdot\left({ }^{1} \chi^{\nu}\right)^{2} .
\end{aligned}
$$

The statistical characteristic of the descriptors included in Model 1 and Model 2 is given in Table 3.

The models obtained [Eqs. (1) and (2)] were used to predict $\log _{10} c m c$ values of compound that were not used in the correlation analysis. The calculated and experimental
Table 1 Correlation coefficient values in first step

Table 2 Correlation coefficient values in second step

Table 3 Statistical characteristic of descriptors included in Models 1 and 2

\begin{tabular}{lllllllllll}
\hline Descriptors & ${ }^{0} \chi$ & ${ }^{1} \chi$ & ${ }^{2} \chi$ & ${ }^{3} \chi_{c}$ & ${ }^{4} \chi_{p c}$ & ${ }^{0} \chi^{v}$ & ${ }^{1} \chi^{v}$ & ${ }^{2} \chi^{v}$ & ${ }^{3} \chi_{c}^{v}$ & ${ }^{4} \chi_{p c}^{v}$ \\
\hline Correlation coefficient & 0.984 & 0.984 & 0.984 & - & - & 0.987 & 0.989 & 0.991 & - & - \\
\hline
\end{tabular}

\begin{tabular}{lllllllllll}
\hline Descriptors & $\left({ }^{0} \chi\right)^{2}$ & $\left({ }^{1} \chi\right)^{2}$ & $\left({ }^{2} \chi\right)^{2}$ & $\left({ }^{3} \chi_{c}\right)^{2}$ & $\left({ }^{4} \chi_{p c}\right)^{2}$ & $\left({ }^{0} \chi^{v}\right)^{2}$ & $\left({ }^{1} \chi^{v}\right)^{2}$ & $\left({ }^{2} \chi^{v}\right)^{2}$ & $\left({ }^{3} \chi_{c}^{v}\right)^{2}$ & $\left({ }^{4} \chi_{p c}^{v}\right)^{2}$ \\
\hline Model 1 & 0.9928 & 0.9929 & 0.9928 & - & - & 0.9934 & 0.9945 & 0.9960 & - & - \\
Model 2 & 0.9941 & 0.9942 & 0.9940 & - & - & 0.9952 & 0.9964 & 0.9961 & - & - \\
\hline
\end{tabular}

\begin{tabular}{llllll}
\hline Model & Descriptor & Coefficient & Standard error & $t$ value & $p$ value \\
\hline 1 & Constant & 10.1484922069 & 1.9414370136 & 5.2273095319 & 0.0012160262 \\
& ${ }^{2} \chi^{v}$ & -1.3905164630 & 0.2822693614 & -4.926204020 & 0.0017015942 \\
& $\left({ }^{2} \chi^{v}\right)^{2}$ & 0.0305970355 & 0.0100815052 & 3.0349669983 & 0.0189811391 \\
2 & Constant & 8.3820502124 & 1.3796130823 & 6.0756528914 & 0.0005030240 \\
& ${ }^{1} \chi^{v}$ & -0.9562437540 & 0.1591621224 & -6.007985691 & 0.0005379592 \\
& $\left({ }^{1} \chi^{v}\right)^{2}$ & 0.0165651687 & 0.0044856720 & 3.6929068104 & 0.0077269162 \\
\hline
\end{tabular}


Table 4 Literature and calculated using Models 1 and $2 \log _{10} c m c$ values of training set compounds 1-10 and test compound $\mathbf{1 1}$

\begin{tabular}{lllrlr}
\hline Compound & $\begin{array}{l}\text { Experimental } \\
\log _{10} c m c \text { [Refer- } \\
\text { ences] }\end{array}$ & $\begin{array}{l}\text { Calculated } \log _{10} c m c \\
\text { Model 1 }\end{array}$ & Residual & $\begin{array}{l}\text { Calculated } \log _{10} c m c \\
\text { Model 2 }\end{array}$ & Residual \\
\hline $\mathbf{1}$ & $-1.22185[24]$ & -1.23848 & 0.01663 & -1.23276 & \\
$\mathbf{2}$ & $-2.04096[24]$ & -2.21642 & 0.17546 & -2.21972 & 0.01091 \\
$\mathbf{3}$ & $-2.95861[24]$ & -3.07198 & 0.11337 & -3.07416 & 0.17876 \\
$\mathbf{4}$ & $-3.82391[25]$ & -3.80515 & -0.01876 & -3.79608 & 0.11555 \\
$\mathbf{5}$ & $-4.30103[24]$ & -4.41593 & 0.11490 & -4.38547 & -0.02783 \\
$\mathbf{6}$ & $-1.79048[26]$ & -1.74275 & -0.04773 & -1.74281 & 0.08444 \\
$\mathbf{7}$ & $-2.29243[26]$ & -2.21642 & -0.07601 & -2.21972 & -0.04767 \\
$\mathbf{8}$ & $-2.74473[26]$ & -2.65950 & -0.08523 & -2.66350 & -0.07271 \\
$\mathbf{9}$ & $-3.18709[26]$ & -3.07198 & -0.11511 & -3.07416 & -0.08123 \\
$\mathbf{1 0}$ & $-4.74473[27]$ & -4.66719 & -0.07754 & -4.69744 & -0.11293 \\
$\mathbf{1 1}$ & $-3.08092[28]$ & -3.07198 & -0.00894 & -3.07416 & -0.04729 \\
\hline
\end{tabular}

$\log _{10} c m c$ values of training set compounds 1-10 along with $\log _{10} c m c$ values of test compound $\mathbf{1 1}$ are listed in Table 4.

The calculated $\log _{10} c m c$ values of training set compounds have been also plotted against the experimental values. The corresponding scatter plots of the experimental $\log _{10} c m c$ values versus the calculated $\log _{10} c m c$ values for Models 1 and 2 are shown in Fig. 1. The plots of residuals versus the experimental values of $\log _{10} \mathrm{cmc}$ are shown in Fig. 2. The $\log _{10} \mathrm{cmc}$ values of additional test compounds 12-14, calculated using both models (Models 1 and 2), are included in Table 5.

Model 2 [Eq. (2)] has been used to examine the variation of $\log _{10} c m c$ with the alkyl chains carbon number $(n)$ for surfactant series $n-6-n$. The corresponding plot of the calculated $\log _{10} \mathrm{cmc}$ versus alkyl chain carbon number of $n-6-n$ compounds for $n=8-28$ is shown in Fig. 3 .

The second-order valence molecular connectivity index $\left({ }^{2} \chi^{v}\right)$, appearing in Model 1, is a path-type index and it represents the two-bond terms in the molecule and its value depends on isomers of the molecule. The value of ${ }^{2} \chi^{v}$ index increases with increasing length and branching of hydrocarbon chains. The first-order valence molecular connectivity index $\left({ }^{1} \chi^{v}\right)$, appearing in Model 2, is a path-type index and it represents the one-bond terms in the molecule. The value of this index increases with increasing hydrocarbon chain length and like the ${ }^{2} \chi^{v}$ index its value depends on isomers but in this case, it decreases with increasing branching $[23,29]$. Of course, when the number of atoms in the molecule increases by adding carbon atoms to the chains through branches, the value of the ${ }^{1} \chi^{v}$ index increases too. Both indices are the valence connectivity indices, therefore, they differentiate multiple bonds.

Therefore, the value of the second-order valence molecular connectivity index and the first-order valence molecular connectivity index increases with increasing length of the alkyl chains and this relationship is a linear relationship. The
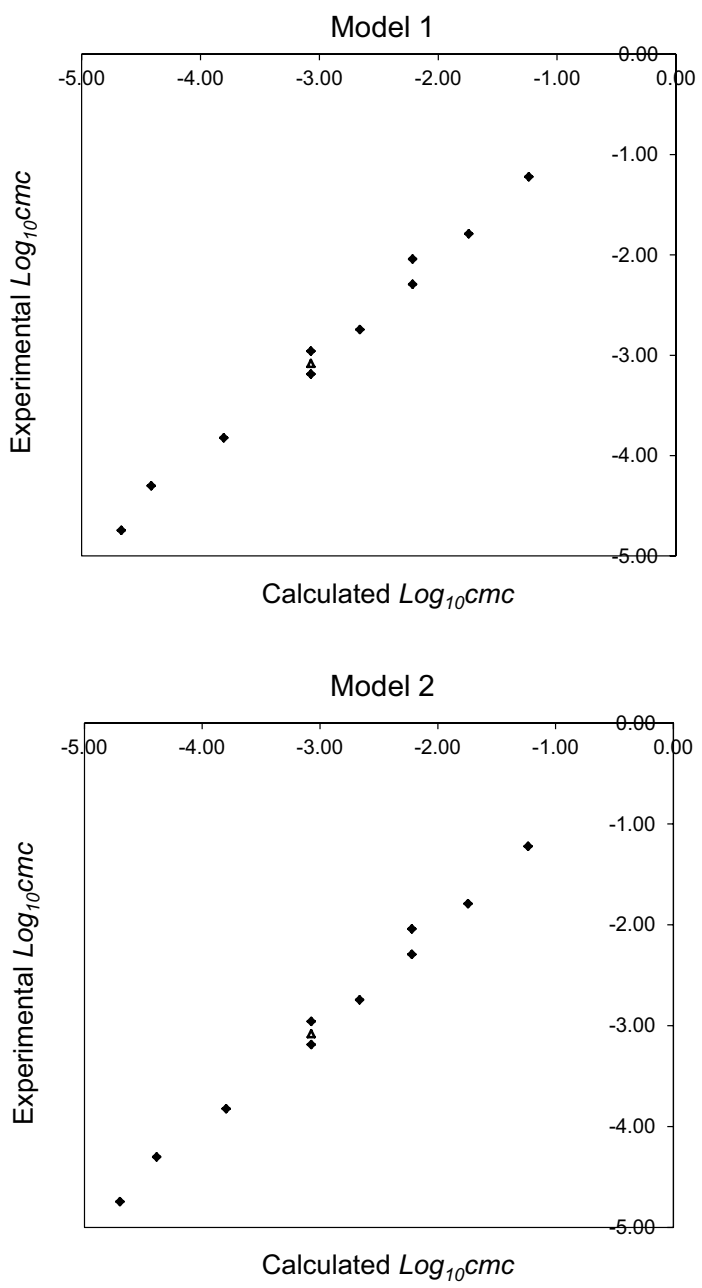

Fig. 1 Plots of the experimental $\log _{10} c m c$ vs. the calculated using Eq. (1) $(r=0.996, F=989.912, s=0.107)$ and Eq. (2) $(r=0.9964$, $F=1105.768, s=0.101)$ for training set (filled diamond) and test compound $\mathbf{1 1}$ (unfilled triangle) 

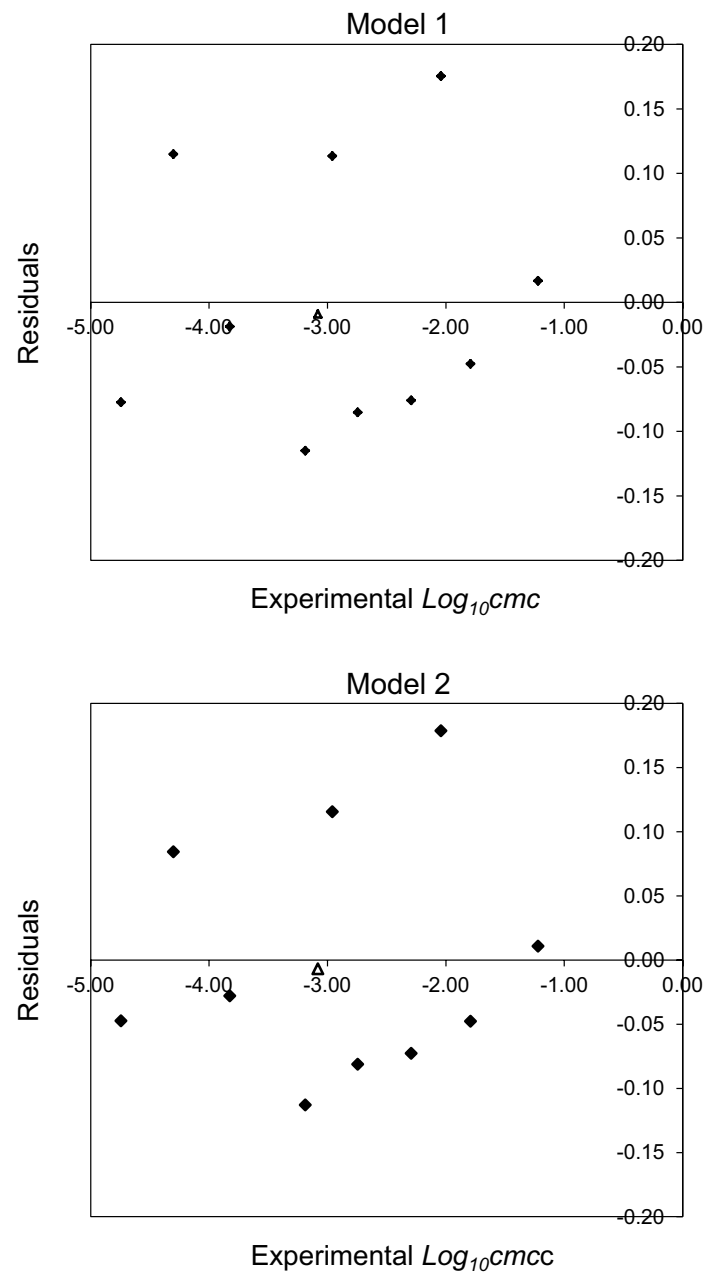

Fig. 2 Plots of the residuals vs. the experimental $\log _{10} \mathrm{Cmc}$ for training set (filled diamond) and test compound $\mathbf{1 1}$ (unfilled triangle)

Table 5 Calculated $\log _{10} \mathrm{Cmc}$ values of compounds 12-14 and compound 3

\begin{tabular}{|c|c|c|}
\hline Compound & $\begin{array}{l}\text { Calculated } \log _{10} c m c \\
\text { Model } 1\end{array}$ & $\begin{array}{l}\text { Calculated } \log _{10} c m c \\
\text { Model } 2\end{array}$ \\
\hline 3 & -3.07198 & -3.07416 \\
\hline 12 & -3.36459 & -2.98974 \\
\hline 13 & -2.64569 & -2.79004 \\
\hline 14 & -2.43948 & -2.27210 \\
\hline
\end{tabular}

equations obtained for surfactants having only hydrocarbon chains [Eqs. (1) and (2)] show that the relationship between $\log _{10} \mathrm{Cmc}$ and the valence molecular connectivity indices of first or second order is parabolic. This suggests that the relationship between $\log _{10} c m c$ and the alkyl chains length is also parabolic.

The experimental data obtained for gemini surfactants with other spacer group confirm that the variation of

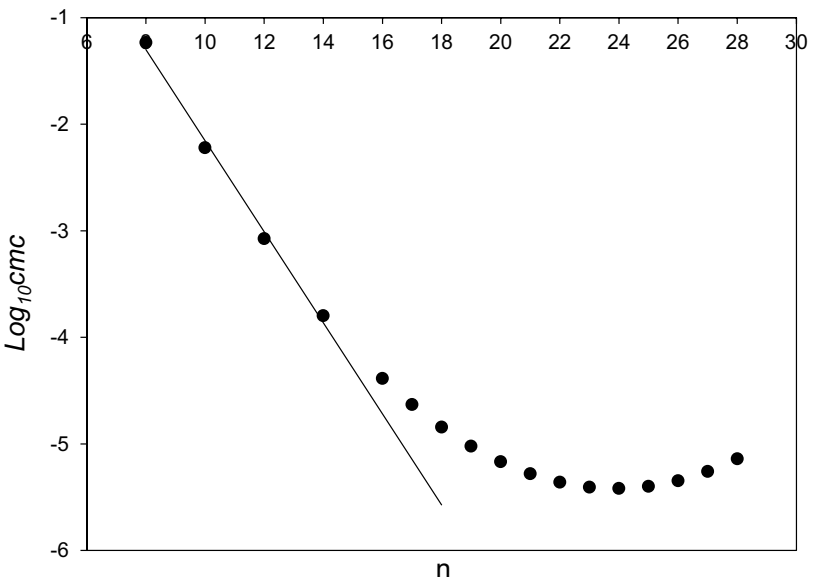

Fig. 3 Calculated using Eq. (2) $\log _{10} c m c$ of $n-6-n$ versus alkyl chain carbon number ( $n$ ) (filled circle)

$\log _{10} \mathrm{cmc}$ with number of carbon atoms in the straight hydrocarbon chains is not linear. The departure from linearity (in water, at $25^{\circ} \mathrm{C}$ ) has been found by Zana [30] for $n-8-n$ gemini surfactants and also by Rosen et al. [19] for gemini surfactants having diethyl ether spacer group. In the latter case, there was observed that the $\log _{10} \mathrm{cmc}$ increases for the alkyl chains length of 20 carbon atoms. In the case of monohydroxypropyl spacer group (in $0.1 \mathrm{~N} \mathrm{NaCl}$, at $25^{\circ} \mathrm{C}$ ) [31], the increase in $\log _{10} \mathrm{cmc}$ value has already been observed for the alkyl chains length of 16 carbon atoms. In these last two cases, this nonlinear dependence seems that can be described by quadratic function.

Models 1 and 2 [Eqs. (1) and (2)] were used to predict the $c m c$ of test compound $\mathbf{1 1}$ and the obtained value using Eq. (1) is $0.847 \mathrm{mmol}$ but using Eq. (2), this value is $0.843 \mathrm{mmol}$, and the experimental value is $0.83 \mathrm{mmol}$ [28]. These and results presented in Tables 3, 4 and Figs. 1, 2 suggest that both models have very good prediction ability of compounds with only hydrocarbon tail chains and Model 2 is slightly better than Model 1.

Both models obtained (Model 1 and 2) have been used to analyse the effect of double bonds, branches and also phenyl groups on $c m c$ value (Table 5). To study these effects, the compounds with 12 carbons atoms in the tail chains and simultaneously with different hydrocarbon tail structures were taken into account (compounds 12-14 in Fig. 4). The comparison of the $\log _{10} \mathrm{cmc}$ value of gemini surfactants with the straight and branched hydrocarbon tail chains (compounds $\mathbf{3}$ and 12, see Table 5), shows that the branches cause the $\mathrm{cmc}$ value calculated using Model 1 to decrease but using Model 2, the branches cause the increase the $\mathrm{cmc}$ value. The result obtained for Model 2 is consistent with the results obtained for monomeric surfactants [1] for which branching results in larger $\mathrm{cmc}$ values in comparison with $\mathrm{cmc}$ values of those having straight 
Fig. 4 Structures of compounds. $R^{1}=\mathrm{C}_{\mathrm{n}} \mathrm{H}_{2 \mathrm{n}+1}(n=8,10,12,14$, 16). $R^{2}=\mathrm{C}_{\mathrm{n}} \mathrm{H}_{2 \mathrm{n}+1}(n=12,14$, $16,18)$
1 - 5<smiles>[R1][N+](C)(C)CCCCCC[N+]([R1])(C)C</smiles>

$6-9$<smiles>[R2][N+](C)(C)CCCCCC[N+](C)(C)C</smiles>

10<smiles>C[N+](C)(C)CCCCCC[N+](C)(C)C</smiles><smiles>C/C=C\CCC</smiles>

11<smiles>CCCCCCCCC[N+](C)(C)C</smiles>

12

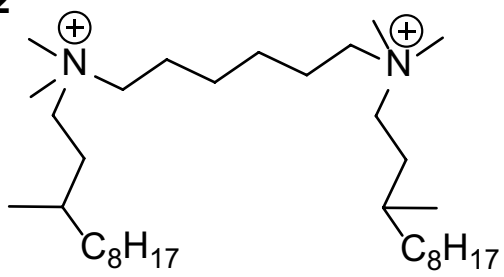

13

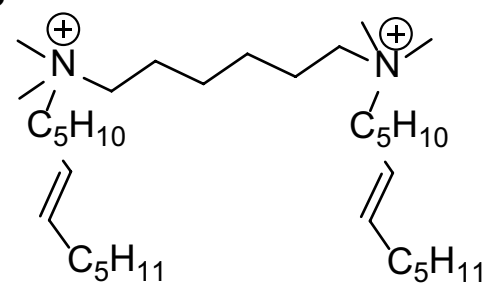

14<smiles>C[N+](C)(CCCCCC[N+](C)(C)c1ccccc1)c1ccccc1</smiles>

chains. As shown in Table 5, the replacement of the single bond with the double one (compounds 3 and 13) causes the increase in $c m c$ value calculated using both models. These results are consistent with those obtained for conventional surfactants [1]. When the phenyl group is present in the hydrophobic tails of the gemini surfactant, the $\mathrm{cmc}$ value is higher than for molecule with the straight tail chains but having the same number of carbon atoms (compounds 3 and 14). These results are also consistent with those obtained for conventional monomeric surfactants [1]. As it was reported in Rosen book [1], the phenyl group is equivalent to about three and one-half methylene groups. The result obtained using Model 2 is approximately consistent with this finding. The calculated $c m c$ value of compound 14 using Eq. (2) is $5.344 \mathrm{mmol}$. This $c m c$ value is slightly lower than that obtained for the gemini surfactant with straight tail chains having ten carbon atoms, for which the calculated $c m c$ value is $6.03 \mathrm{mmol}$.

Above results show that Model 2 [Eq. (2)] better predicts and describes changes in values of the critical micelle concentration. Thus, Model 2 can be considered as the best.

The best model (Model 2) has been used to examine the variation of $\log _{10} \mathrm{Cmc}$ with number of alkyl chains carbon atoms ( $n$ ) of $n-6-n$ compounds and also to predict the minimum $\mathrm{cmc}$ for those compounds. The plot of the calculated $\log _{10} \mathrm{cmc}$ versus carbon number from $n=8$ to $n=28$ is presented in Fig. 3. As can be seen in the Fig. 3, a straight line can be drawn through the first four points and from $n=16$, the $\log _{10} c m c$ with further increase of $\mathrm{n}$ begins to deviate from that line reaching the minimum of $n=24$. Thus, as shown in Fig. 3, the smallest $c m c$ value has the compound having hydrocarbon chains with 24 carbon atoms and is equal to $0.0038 \mathrm{mmol}$. This result seems to be consistent with reality, or at least approximately.

\section{Conclusion}

The results obtained in this work using Eq. (2) are in good agreement with the experimental ones, including those results that relate to compounds not used in the correlation analysis. Model 2 (Eq. (2)) very good describes and predicts critical micelle concentration values of compounds having the quaternary ammonium head groups, the spacer group of medium length and the hydrocarbon tail chains. The present theoretical studies on gemini surfactant hydrophobic tail chains suggest that the dependence of $\log _{10} \mathrm{Cmc}$ on the alkyl chains length is nonlinear, and the obtained equation [Eq. (2)] shows that it is the parabolic relationship. This suggests that at some tail chains length, even when lengthening the hydrocarbon only chains, the $c m c$ values will start to increase. 
Table 6 Experimental $\mathrm{cmc}$ values and molecular connectivity indices values of training set compounds

\begin{tabular}{|c|c|c|c|c|c|c|c|c|c|c|c|c|}
\hline $\begin{array}{l}\text { Com- } \\
\text { pound }\end{array}$ & $\begin{array}{l}\text { cmca } \\
\text { (mmol) } \\
\text { [References] }\end{array}$ & ${ }^{0} \chi$ & ${ }^{1} \chi$ & ${ }^{2} \chi$ & ${ }^{3} \chi_{c}$ & ${ }^{4} \chi_{p c}$ & ${ }^{0} \chi^{v}$ & ${ }^{1} \chi^{v}$ & ${ }^{2} \chi^{v}$ & ${ }^{3} \chi_{c}^{v}$ & ${ }^{4} \chi_{p c}^{v}$ & [References] \\
\hline 1 & $\begin{array}{l}60 \\
{[24]}\end{array}$ & 21.14214 & 13.32843 & 11.27817 & 2.41421 & 2.41421 & 21.03656 & 12.96798 & 10.71564 & 2.15934 & 2.15934 & [7] \\
\hline 2 & $\begin{array}{l}9.1 \\
{[24]}\end{array}$ & 23.97057 & 15.32843 & 12.69238 & 2.41421 & 2.41421 & 23.86499 & 14.96798 & 12.12985 & 2.15934 & 2.15934 & [7] \\
\hline 3 & $\begin{array}{l}1.1 \\
{[24]}\end{array}$ & 26.79899 & 17.32843 & 14.10660 & 2.41421 & 2.41421 & 26.69341 & 16.96798 & 13.54407 & 2.15934 & 2.15934 & [7] \\
\hline 4 & $\begin{array}{l}0.15 \\
{[25]}\end{array}$ & 29.62742 & 19.32843 & 15.52081 & 2.41421 & 2.41421 & 29.52184 & 18.96798 & 14.95828 & 2.15934 & 2.15934 & [7] \\
\hline 5 & $\begin{array}{l}0.05 \\
{[24]}\end{array}$ & 32.45585 & 21.32843 & 16.93502 & 2.41421 & 2.41421 & 32.35027 & 20.96798 & 16.37249 & 2.15934 & 2.15934 & [7] \\
\hline 6 & $\begin{array}{l}16.2 \\
{[26]}\end{array}$ & 22.55635 & 14.32843 & 11.98528 & 2.41421 & 2.41421 & 22.45078 & 13.96798 & 11.42275 & 2.15934 & 2.15934 & [7] \\
\hline 7 & $\begin{array}{l}5.1 \\
{[26]}\end{array}$ & 23.97057 & 15.32843 & 12.69238 & 2.41421 & 2.41421 & 23.86499 & 14.96798 & 12.12985 & 2.15934 & 2.15934 & [7] \\
\hline 8 & $\begin{array}{l}1.8 \\
{[26]}\end{array}$ & 25.38478 & 16.32843 & 13.39949 & 2.41421 & 2.41421 & 25.2792 & 15.96798 & 12.83695 & 2.15934 & 2.15934 & [7] \\
\hline 9 & $\begin{array}{l}0.65 \\
{[26]}\end{array}$ & 26.79899 & 17.32843 & 14.10660 & 2.41421 & 2.41421 & 26.69341 & 16.96798 & 13.54407 & 2.15934 & 2.15934 & This work \\
\hline 10 & $\begin{array}{l}0.018 \\
{[27]}\end{array}$ & 35.28427 & 23.32843 & 18.34924 & 2.41421 & 2.41421 & 34.65967 & 22.26764 & 17.05579 & 2.15934 & 2.15934 & This work \\
\hline
\end{tabular}

${ }^{\text {a }}$ All values of $c m c$ were measured in pure water at $25^{\circ} \mathrm{C}$ by conductivity method

\section{Methods}

The entire data set contains gemini surfactants with only the hydrocarbon tail chains, having the quaternary ammonium head groups connected by the spacer group of six carbon atoms length. All investigated gemini surfactants have the bromides as counterions. The structures of all considered compounds are shown in Fig. 4.

The training set contains the gemini surfactants with tail chains of varying lengths and flexibility. The test set contains one compound having tail chains of different lengths and also the surfactants with tails with fixed number of carbon atoms equal to 12 carbons but differing in structure. Thus, the data set includes ten compounds of training set (compounds 1-10) and four compounds of test set (compound $\mathbf{1 1}$ and additional compounds 12-14). The chemical structures of compounds of training set and test compound 11, and the experimental values of $\mathrm{cmc}$ were taken from the literature [24-28].

The Kier and Hall molecular connectivity indices [23] were calculated based on the graphic structural formula of the molecule [7]. The values of molecular connectivity indices and the $c m c$ values of compounds from training set are listed in Table 6.

Each formula expressing the relationship between $\log _{10} \mathrm{Cmc}$ and the molecular connectivity indices was generated using the least-squares method. The final equation was obtained using the stepwise method. The quality of the derived models was tested using the following statistical parameters: the correlation coefficient $(r)$, the Fisher ratio $(F)$, and the standard deviation of the fit $(s)$. The values of the correlation coefficient closer to 1 represent the better fit of the model. The larger the value of the Fisher ratio and the smaller the standard deviation indicate that the model is statistically significant.

High absolute Student $t$ value of the descriptors, in statistical characteristic of the descriptors (Table 3), express that the regression coefficients of the descriptors are significantly larger than the standard error. The descriptors with the $p$ values below 0.05 are considered statistically significant.

All statistical calculations were performed using the program STATISTICA 12 [32].

Acknowledgements The statistical calculations were performed using the program Statistica 12 provided by the Wrocław University of Environmental and Life Sciences.

Open Access This article is licensed under a Creative Commons Attribution 4.0 International License, which permits use, sharing, adaptation, distribution and reproduction in any medium or format, as long as you give appropriate credit to the original author(s) and the source, provide a link to the Creative Commons licence, and indicate if changes were made. The images or other third party material in this article are included in the article's Creative Commons licence, unless indicated otherwise in a credit line to the material. If material is not included in the article's Creative Commons licence and your intended use is not permitted by statutory regulation or exceeds the permitted use, you will 
need to obtain permission directly from the copyright holder. To view a copy of this licence, visit http://creativecommons.org/licenses/by/4.0/.

\section{References}

1. Rosen MJ (2004) Surfactants and interfacial phenomena. Wiley, New Jersey

2. Mozrzymas A (2019) J Solut Chem 48:875

3. Baghban A, Sasanipour J, Sarafbidabad M, Piri A, Razavi R (2018) Chem Phys Lipids 214:46

4. Mozrzymas A (2017) Colloid Polym Sci 295:75

5. Mozrzymas A (2016) Comb Chem High Throughput Screen 19:481

6. Zhu ZC, Wang Q, Jia QZ, Tang HM, Ma PS (2013) Acta PhysChim Sin 29:30

7. Mozrzymas A (2013) J Solut Chem 42:2187

8. Roy K, Kabir H (2012) Chem Eng Sci 73:86

9. Roy K, Kabir H (2012) Chem Eng Sci 81:169

10. Mozrzymas A, Różycka-Roszak B (2011) J Math Chem 49:276

11. Mozrzymas A, Różycka-Roszak B (2010) Comb Chem High Throughput Screen 13:39

12. Stanton DT (2008) J Comput Aided Mol Des 22:441

13. Gad EAM (2007) J Dispers Sci Technol 28:231

14. Kardanpour Z, Hemmateenejad B, Khayamian T (2005) Anal Chim Acta 531:285

15. Absalan G, Hemmateenejad B, Soleimani M, Akhond M, Miri R (2004) QSAR Comb Sci 23:416

16. Yuan S, Cai Z, Xu G, Jiang Y (2002) J Dispers Sci Technol 23:465
17. Wang Z, Li G, Zang X, Wang R, Lou A (2002) Colloids Surf A 197:37

18. Huibers PDT, Lobanov VS, Katritzky AR, Shah DO, Karelson M (1996) Langmuir 12:1462

19. Klevens HB (1953) J Am Oil Chem Soc 30:74

20. Rosen MJ, Mathias JH, Davenport L (1999) Langmuir 15:7340

21. Taleb K, Mohamed-Benkada M, Benhamed N, Saidi-Besbes S, Grohens Y, Derdour A (2017) J Mol Liquids 241:81

22. Ghumare AK, Pawar BV, Bhagwat SS (2013) J Surf Deterg 16:85

23. Kier LB, Hall LH (1986) Molecular Connectivity in structureactivity analysis. Research Studies Press Ltd, Letchworth

24. Junior PBS, Tiera VAO, Tiera MJ (2007) Ecletica Quim 32:47

25. Ali MS, Suhail M, Ghosh G, Kamil M, Kabir-ud-Din (2009) Colloids Surf A 350:51

26. Fan Y, Li Y, Cao M, Wang J, Wang Y, Thomas RK (2007) Langmuir 23:11458

27. Li X, Wettig SD, Wang C, Foldvari M, Verrall RE (2005) Phys Chem Chem Phys 7:3172

28. Wang X, Wang J, Wang Y, Ye J, Yan H, Thomas RK (2003) J Phys Chem B 107:11428

29. Contrera JF, MacLaughlin P, Hall LH, Kier LB (2005) Curr Drug Discov Technol 2:55

30. Zana R (2002) J Colloid Interface Sci 246:182

31. Song LD, Rosen MJ (1996) Langmuir 12:1149

32. STATISTICA (data analysis software system) (2014) version 12, StatSoft, Inc.; software. https://www.statsoft.com

Publisher's Note Springer Nature remains neutral with regard to jurisdictional claims in published maps and institutional affiliations. 